\title{
Investigation Methods for Power Quality Problems in Large Scale LV Network
}

\author{
P. R. Wilczek, M. C. Benhabib, J. M. A. Myrzik, J. L. Duarte \\ Department of electrical engineering, Eindhoven University of Technology \\ Den Dolech 2, CR 0.03, P.O. Box 513, 5600 MB Eindhoven, The Netherlands \\ Tel.: 003140247 2376, Fax: 0031402450735 \\ P.Wilczek@tue.nl, M.C.Benhabib@tue.nl, J.M.A.Myrzik@tue.nl, J.L.Duarte@tue.nl
}

\begin{abstract}
Nowadays the number of nonlinear loads and dispersed generators connected to the LV network increases. The influence of nonlinear devices, like energy saving lamps, computers or photovoltaic inverters on power quality in the grid has to be investigated. Analysis of a large number of nonlinear devices in complex network is difficult since every device has to be simulated with the complete model. For this reason a new method is developed. In this method the complete model of a device is replaced by an equivalent reduced model. Since the model is simple, a large number of the devices can be simulated.

In this paper an improved equivalent reduced model is presented. The improved model allows simulations of the behavior of the devices when a dip or swell occurs. Three nonlinear devices are measured and modeled. Experimental and simulation results are presented.
\end{abstract}

Key words - harmonic interaction, equivalent reduced model, nonlinear devices, voltage variation.

\section{Introduction}

A simulation of a low voltage network, containing several nonlinear loads and dispersed generators such as PV systems, can be performed in two ways.

The first consists in carrying out a complete model of each nonlinear load and/or dispersed generation, in order to generate all the harmonics injected by this type of equipment. This delivers the most accurate results but it is not straightforward owing to the fact that the memory of a powerful computer is running out because of complexity of the LV network.

At the second, a simplified model can be used. If the model of each equipment, like a nonlinear load or photovoltaic generator is simplified, a loss of information concerning the harmonics spectrum and phases can be obtained.

To solve this problem the authors have presented in [1] and [2] a new way to study this kind of complex systems. In this method the equipment is represented by a simple equivalent circuit that replaces the complete model of the equipment. This model consists of a current source and impedance connected in parallel. This model gives a very good result for studying harmonics interaction and resonance problems. However, concerning the problem of dips or swells, this model must be improved. This is what the authors artes: going 10 o present in this paper.

\section{Description of the Reduced Model}

The basic goal of this method given by [1] and [2] is to build a reduced model of a device. The model consists of a programmable current source representing the harmonic current generated by the device. The input data for the current source is taken from the complete model simulated or from an experimental branch. In parallel to the source a capacitor or inductor is connected, as shown on Figure 1, representing capacitance or inductance insertion to the grid.

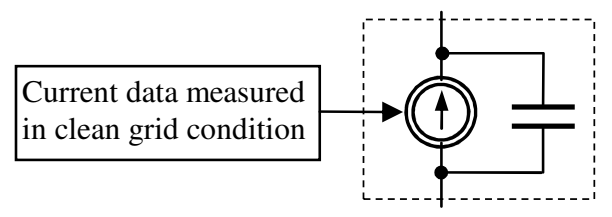

Fig. 1. Reduced equivalent model of equipment that consist a current source in parallel with capacitor.

The model of the used devices is build on the basis of a series of measurements. First, the current of the equipment is measured in clean grid at nominal voltage level. This data will be used in simulation as input for the current source. Next, harmonic voltage is generated by a grid simulator and harmonic current is measured. The value of the capacitance (or inductance) is calculated on the basis of magnitude and angle of the measured current and voltage. Several measurements are performed at different frequencies. The frequencies must be free of harmonic currents generated by the device (measured in clean grid) or these currents must be taken into account during calculations.

\section{Improved Reduced Model for Voltage Variation}

The improved model introduces a link between the voltage at the device's terminals and the current of the device as shown on Figure 2. This link makes it possible to simulate the effect of a dip or swell on the output or input current of the device. 


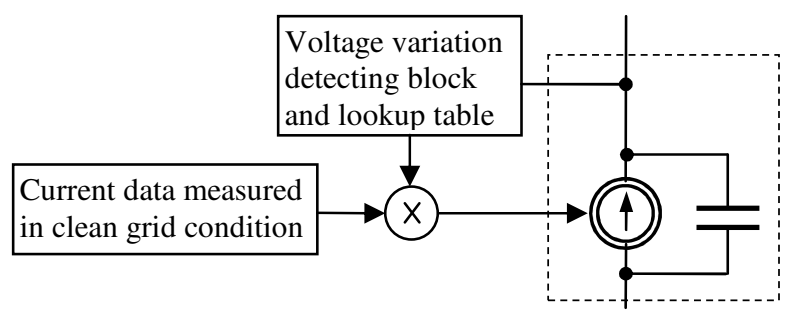

Fig. 2. Improved equivalent model with voltage variation block

The current value will be increased or decreased depending on the voltage on the device's terminals according to a lookup table. This sinusoidal voltage is sampled at every peak during the simulation. To detect the peak, the voltage signal is shifted 90 degrees by using the derivative of the voltage. The shifted signal is connected to a zero crossing block that gives an impulse at the zero of shifted signal and consequently at the voltage peak. The impulse triggers a sample and hold block to sample the voltage and keep constant until the next voltage peak.

The voltage peak value is used as input for the look-up table. The second input is the current data that is multiplied depending on the voltage value and according to the table data. As a result the current is increased or decreased in case of a dip or swell.

To provide the data for the look-up table a series of measurements is necessary. The current of the device to be simu- lated, has to be measured at several voltage levels ex: 70, 80, $90,100,105$ and $110 \%$ of the nominal voltage.

Since the derivative is used to shift the voltage signal, it's important that the voltage data, esp. when taken from an experiment, is free of noise. That imposes on us to use a good filter without giving any additional shift. A multivariable band past filter proposed by [3] and [4] is used. The details of this filter are proposed in the same paper [3] and [4].

Remarque: The filter is used when the voltage is transformed from a-b-c axis (three-phase) to $\alpha-\beta$ axis (two-phase). But because only one single phase signal is used, it is necessary to shift the phase a by $90^{\circ}$ by use a $90^{\circ}$ shift method or Hilbert filter.

The terminals voltage is split into two signals. One is taken directly and the other is shifted by $90^{\circ}$. The equations of this filter followed from [3] and [4] are given as:

$$
\begin{gathered}
\hat{\mathrm{x}}_{\alpha}(\mathrm{s})=\frac{\mathrm{K}}{\mathrm{s}}\left[\mathrm{x}_{\alpha}(\mathrm{s})-\hat{\mathrm{x}}_{\alpha}(\mathrm{s})\right]-\frac{\omega_{\mathrm{c}}}{\mathrm{s}} \hat{\mathrm{x}}_{\beta}(\mathrm{s}) \\
\hat{\mathrm{x}}_{\beta}(\mathrm{s})=\frac{\mathrm{K}}{\mathrm{s}}\left[\mathrm{x}_{\beta}(\mathrm{s})-\hat{\mathrm{x}}_{\beta}(\mathrm{s})\right]+\frac{\omega_{\mathrm{c}}}{\mathrm{s}} \hat{\mathrm{x}}_{\alpha}(\mathrm{s})
\end{gathered}
$$

The Figure 4 shows the block diagram of the multivariable filter.

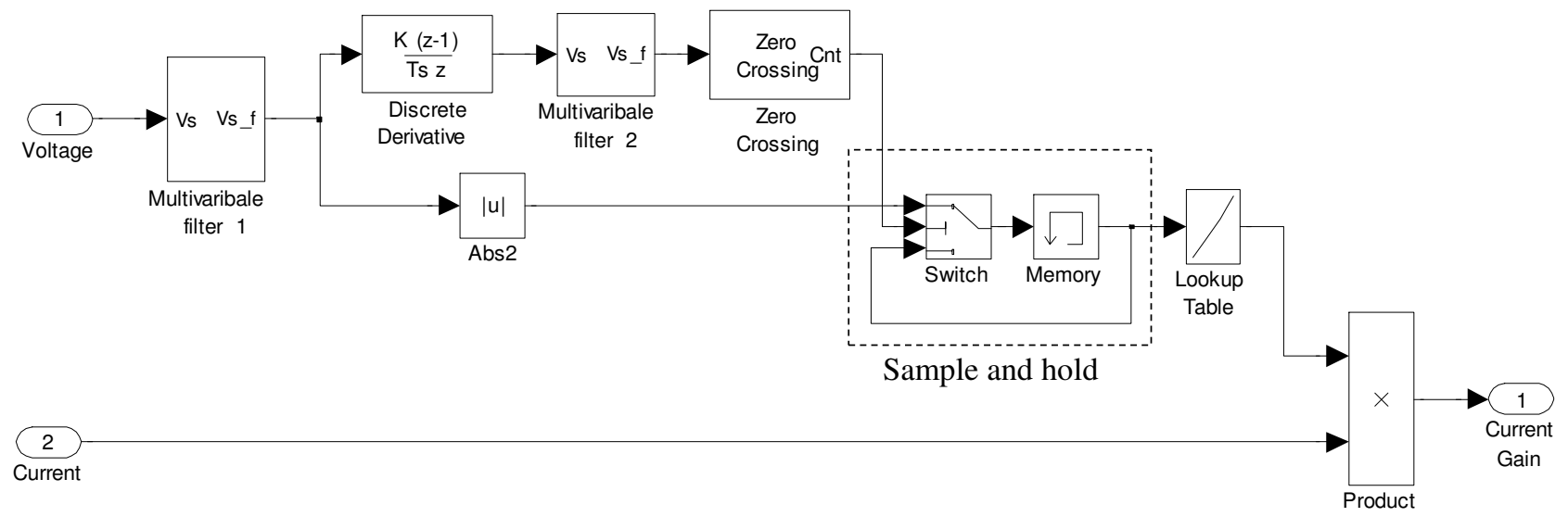

Fig. 3. Voltage variation detecting block and lookup table

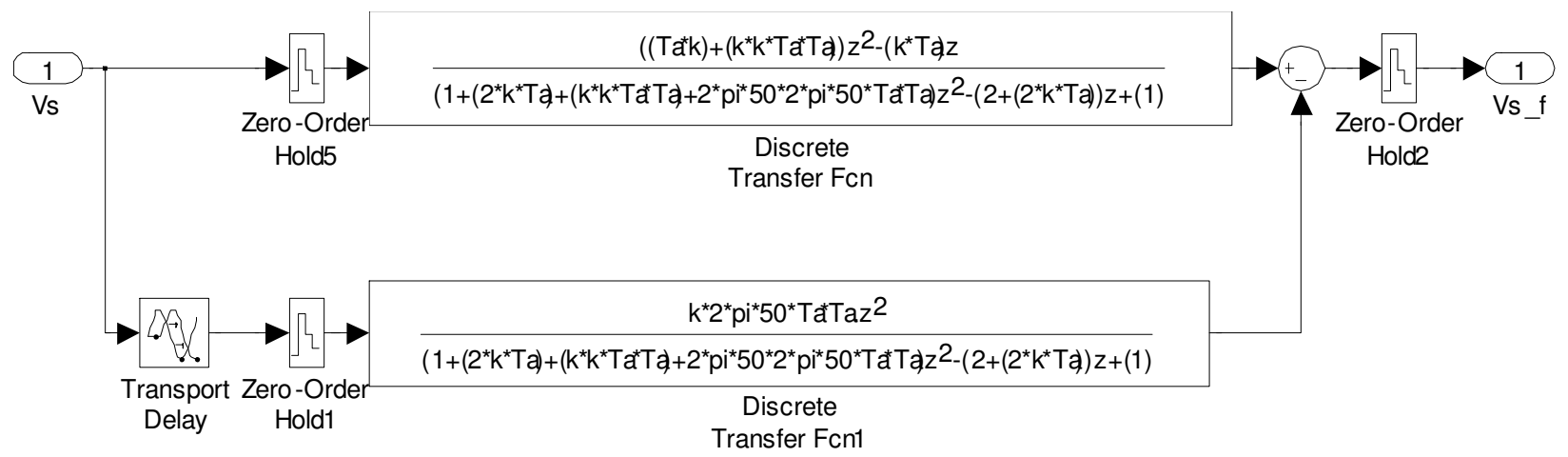

Fig.4. Block diagram of multivariable filter. 


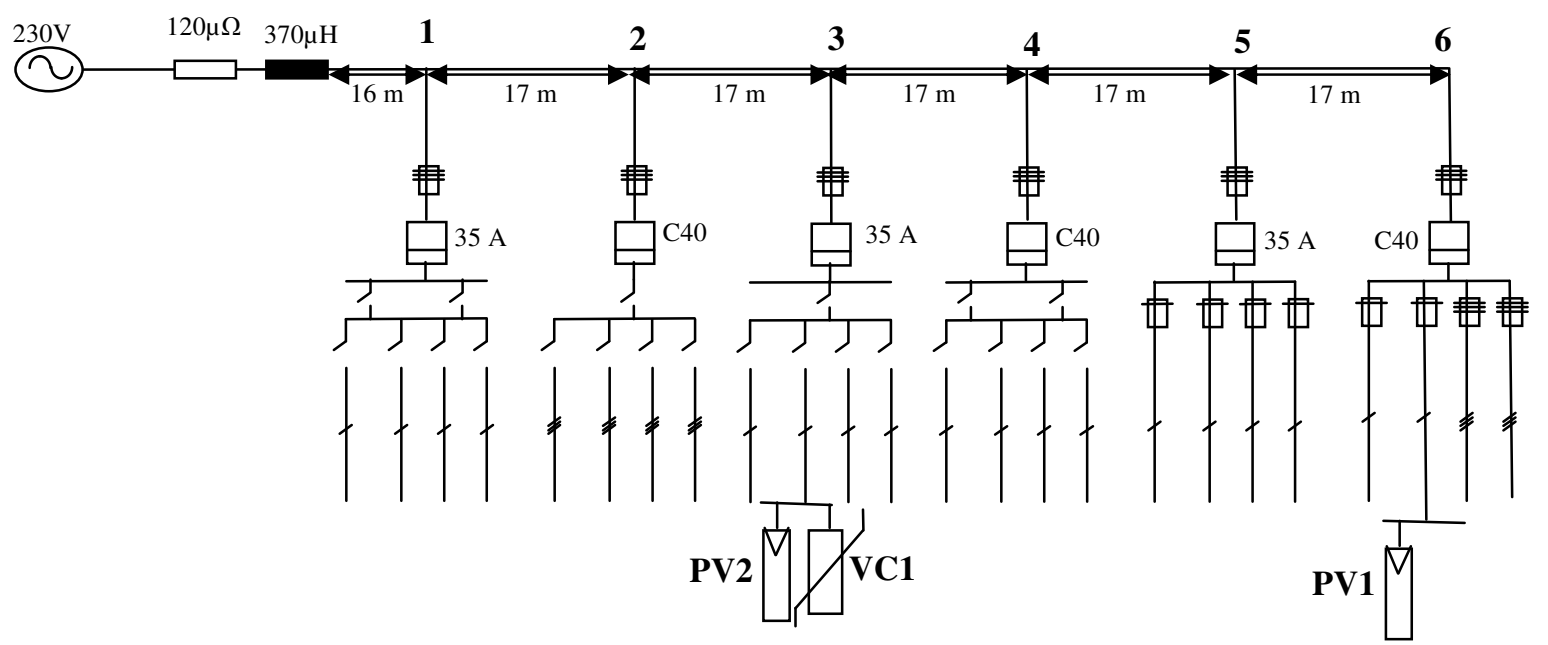

Fig. 5. Experimental LV network

\section{Experimental Validation}

The theoretical equivalent model is validated by comparison of the simulation results and the experimental measurements. Three devices are tested: two photovoltaic inverters and a nonlinear load. The first PV inverter (PV1) is transformerless topology and the other (PV2) is $50 \mathrm{~Hz}$ transformer topology. Each inverter is connected to a solar cell emulator with the maximum power point equal to $1.2 \mathrm{~kW}$. The nonlinear load is a vacuum cleaner with a power electronic circuit that gives highly distorted current.

The experiments are performed in the LV network shown on Figure 5. The devices are connected to the same phase of the network. The currents and voltages are recorded and compared with the simulation results.

There are four scenarios considered. Firstly each device is tested separately, then all together. In all the scenarios the same dip of $80 \%$ of the nominal voltage for $100 \mathrm{~ms}$ is created.

In the first scenario only one photovoltaic inverter (PV1) is connected to the feeder no. 6 of the network shown on Figure 5. The dip is created and the current and voltage of the inverter is recorded (Figure 6).

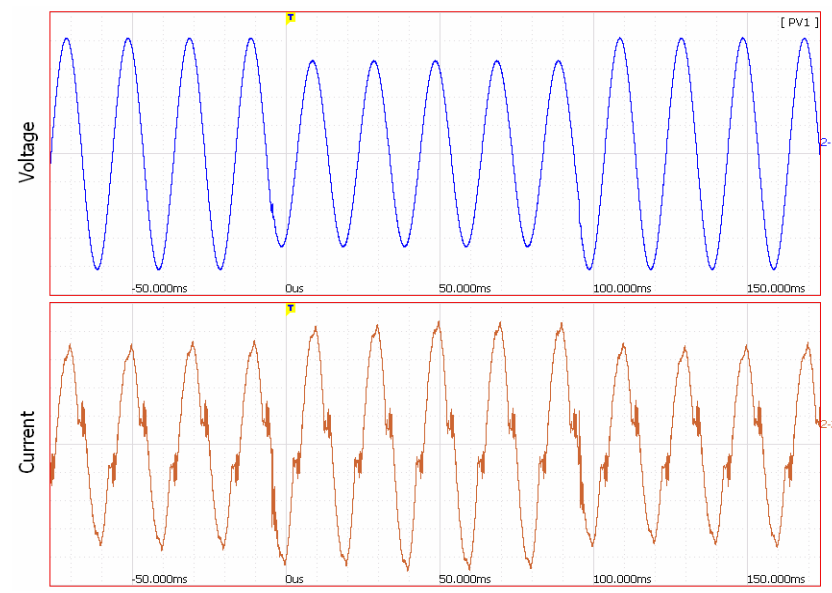

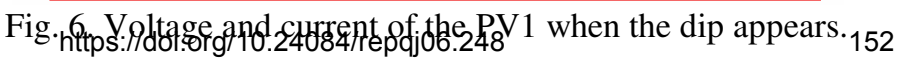

How shown on this figure, the current value of the inverter increases during the dip. It seems that the inverter tries to keep the output power constant despite the voltage level.

To run the simulation, the current of the PV1 at nominal voltage is taken and the same voltage dip is created. The simulation results are given in the Figure 7.
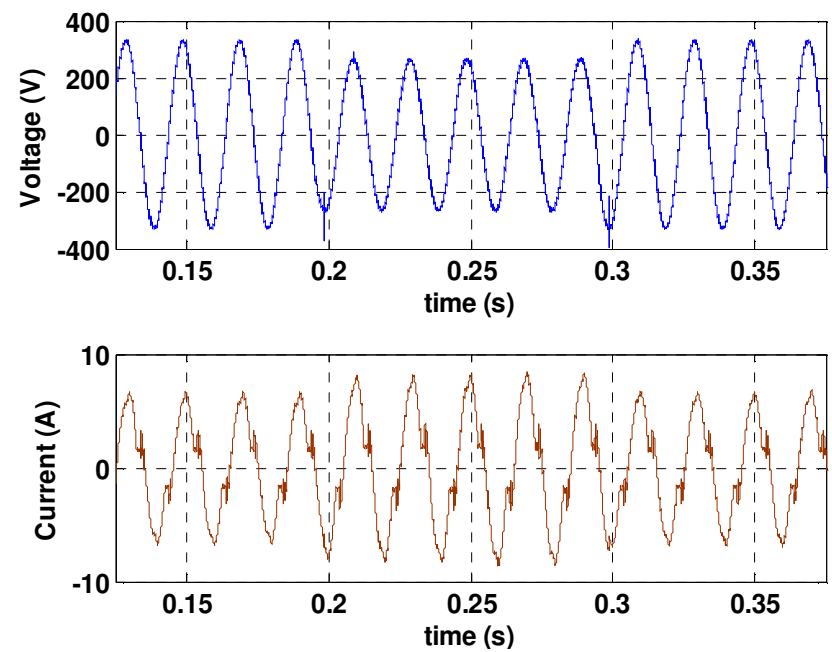

Fig. 7. Simulated voltage and current of the PV1 when dip appears.

As shown on the above figure, the simulation results are the same as in the experiment. The same current increase can be observed.

In the second scenario the PV1 system is disconnected from the feeder no. 6 and the other PV inverter (PV2) is connected to the feeder 3 . The same dip is created and the voltage and current recorded. The test results are shown on Figure 8. 


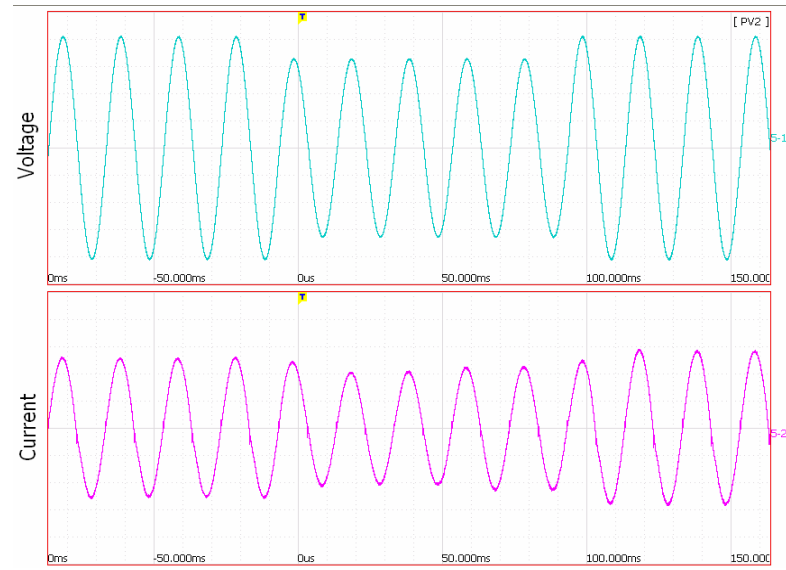

Fig. 8. Experimental voltage and current of the PV2 at dip

Behavior of this inverter is different from the previous one. The current decreases during the dip resulting in lower generated power.

The simulation is performed with the current data of the PV2 taken from the experiment at rated voltage level. The same result is obtained as given on the figure 9 .
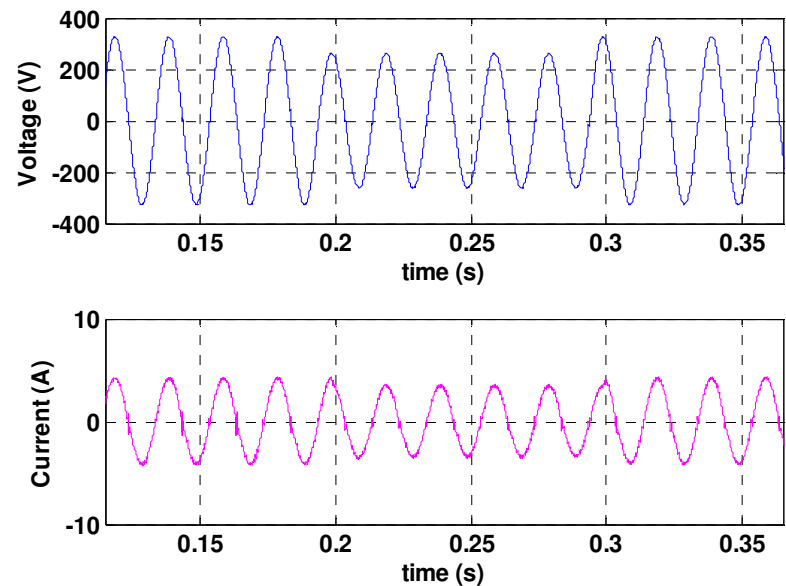

Fig. 9. Simulated voltage and current of the PV2 at dip

In the third scenario only the nonlinear load $\mathrm{VC} 1$, is connected to the feeder 3 . The PV1 and PV2 systems are disconnected from the network. The current and voltage obtained from the experimental branch are given on the Figure 10.

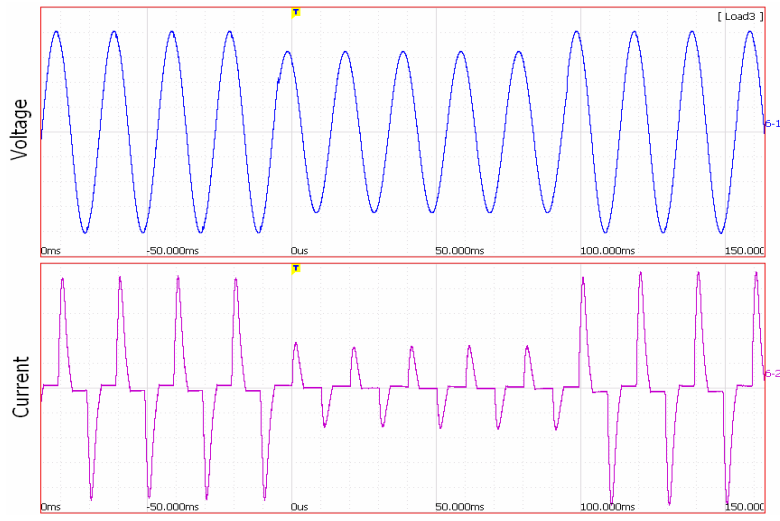

Fig. 10. Experimental voltage and current of the VC1 when

the hipps.ppear.org/10.24084/repqj06.248
The simulation is performed in the same way and results are shown on Figure 11.
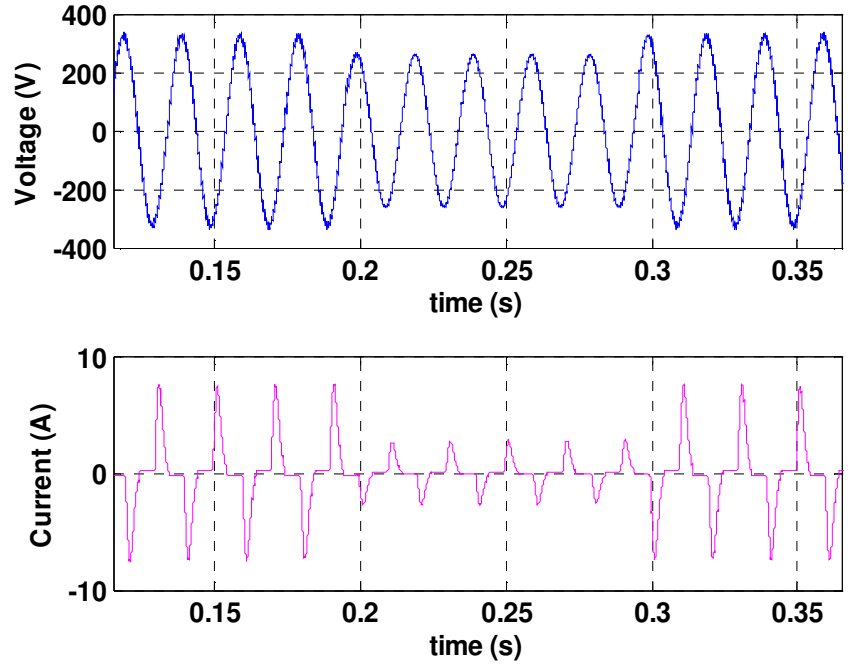

Fig. 11. Simulated voltage and current of the VC1 at dip

These three scenarios were important to do them separately to demonstrate how each nonlinear device reacts when a voltage dip appears. Now the combination of the equipment will be investigated.

In the fourth scenario all the devices are connected the network at the same time. The inverter PV1 is connected to the feeder 6 and inverter PV2 with the load VC1 to the feeder 3 as shown on Figure 5. The dip is created and the current and voltage of each device, as well as the total current, are recorded and presented on figure 12.

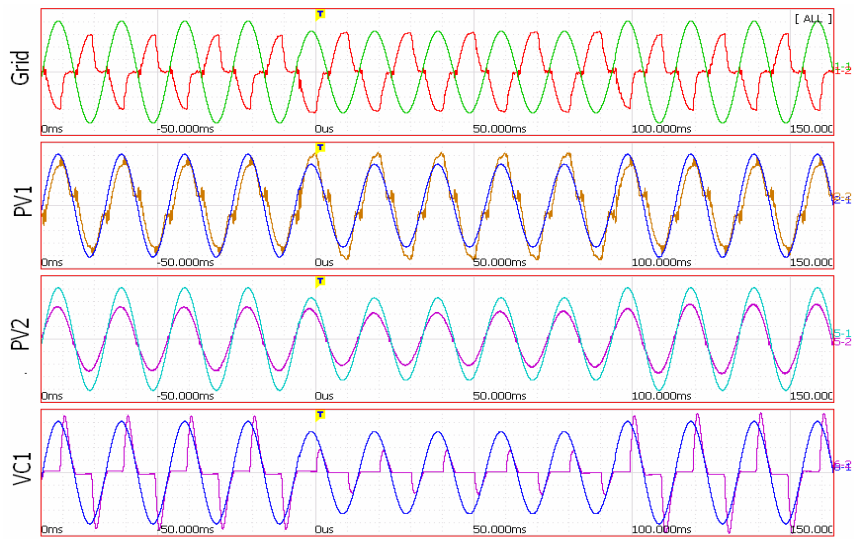

Fig. 12. Experimental voltage and current of grid, PV1, PV2 and $\mathrm{VC} 1$

The simulation results are shown on Figure 13. Each subplot shows voltage and current of the grid, PV1, PV2 and $\mathrm{VC} 1$ respectively. The grid current is in opposite phase to the voltage, meaning that it's feeding the grid. The simulated and experimental total currents are the same. 

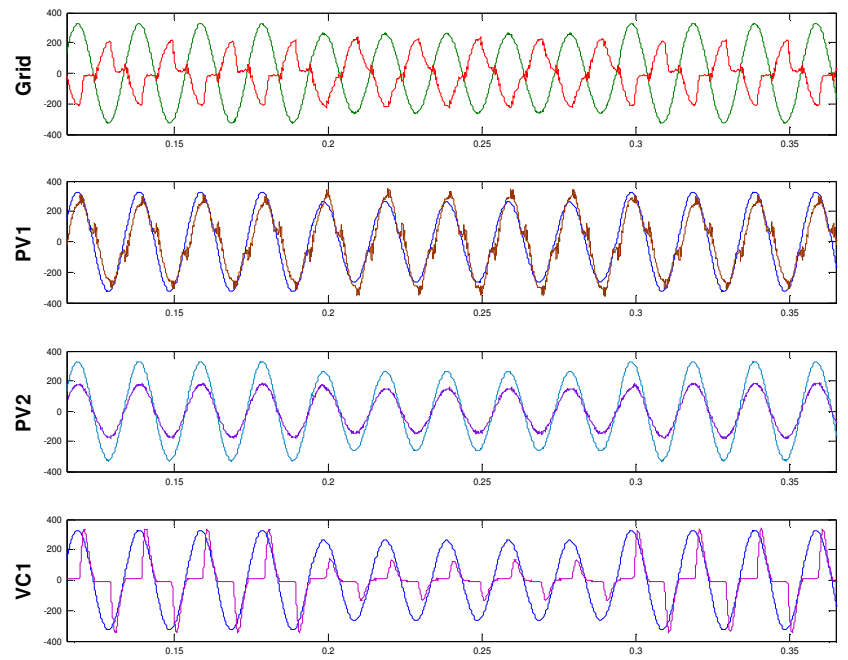

Fig. 13. Simulated voltage and current of grid, PV1, PV2 and $\mathrm{VC} 1$

\section{Conclusions}

The presented reduced equivalent model is simple but gives very accurate results. The model introduces a link between voltage and current of the device. It can be used for studying the behavior of a large amount of nonlinear loads or dispersed generation in the complex LV network. Together with the research on harmonics demonstrated in [1], [2] it can be concluded that this reduced model works very well in a dip or swell condition as well as harmonic interaction of the equipment.

\section{References}

[1] M. C. Benhabib, J. M. A. Myrzik, J. L. Duarte "Harmonics Effect caused by Large Scale PV Installations in a LV Network", EPQU conference Spain 2007.

[2] M. C. Benhabib, P. R. Wilczek, J. M. A. Myrzik, J. L. Duarte "Harmonic interactions and resonance problems in large scale LV networks", submitted to PSCC conference 2008.

[3] M.C Benhabib and S. Saadate "A New Robust Experimentally Validated Phase Locked Loop for Power Electronic Control" 2005, VOL 15; NUMB 3, pages 36-48, EPE JOURNAL.

[4] M.C Benhabib "Contribution à l'étude des différentes topologies et commandes des filtres actifs parallèles à structure tension : Modélisation, simulation et validation expérimentale de la commande" $\mathrm{PhD}$ thesis at University Henri Poincare, Nancy-I, 2004. 